\title{
Valgus deformity and proximal subluxation of the rheumatoid elbow: a radiographic 15 year follow up study of 148 elbows
}

\author{
J T Lehtinen, K Kaarela, M J Kauppi, E A Belt, H Mäenpää, P Kuusela, M U K Lehto
}

\begin{abstract}
Objective-To evaluate the nature of positional changes of humeroulnar (HU) and humeroradial (HR) joints in a cohort of 74 patients with seropositive and erosive rheumatoid arthritis (RA) followed up prospectively.
\end{abstract}

Methods-At the 15 year follow up standard anteroposterior and lateral radiographs of 148 elbow joints were evaluated. The mediolateral HU angle of the elbow was measured from anteroposterior radiographs. The proximal subluxation of the HU joint was measured from lateral radiographs as the distance between the posterior aspect of the olecranon process and the posterior surface of the humerus. The anteroposterior subluxation of the HR joint was measured from lateral radiographs as the relation of the midpoint of head of the radius to the midpoint of the capitellum of the humerus. Destruction of the elbow joints was assessed with the Larsen method on a scale of 0 to 5 and compared with the measurements.

Results-Mean HU angle in 148 elbows of patients with $R A$ was $11.5^{\circ}$ (SD 6.1), range $-21^{\circ}$ (varus) to $34^{\circ}$ (valgus); $9.9^{\circ}$ (SD 4.3) in men and $12.0^{\circ}$ (SD 6.4) in women. The mean HU angle, $14.4^{\circ}$ (SD 6.0) of the affected joints (Larsen grades 2-4), showed more valgus than the mean $9.8^{\circ}$ (SD 2.5) of the non-affected (Larsen grades 0 to 1 ) joints; totally destroyed and unstable Larsen 5 joints were excluded. Mean HU and HR subluxations, $2.0 \mathrm{~mm}$ (SD 3.8) and $0.8 \mathrm{~mm}$, of the affected joints (Larsen 2-5) were greater than the means, $-1.1 \mathrm{~mm}$ (SD 1.5) and $-0.4 \mathrm{~mm}$ (SD 0.9), of the non-affected joints. Both the HU proximal subluxation and the $H R$ anterior subluxation correlated, $r_{\mathrm{s}}=0.64(95 \%$ CI 0.53 to 0.73 ) and $r_{\mathrm{s}}=0.48(95 \%$ CI 0.34 to 0.60 ), with the destruction of the elbow joint.

Conclusions-The elbow seems to turn into valgus during rheumatoid destruction and excision of the radial head may speed up this process. However, totally unstable Larsen grade 5 joints may also have varus deformity owing to mutilating bone destruction. The ulna subluxates proximally in relation to the humerus, whereas the radius moves slightly anteriorly as a consequence of elbow involvement.

(Ann Rheum Dis 2001;60:765-769)
The elbow joint is composed of three bones: the humerus, the radius, and the ulna. The humeroulnar (HU) articulation permits flexion and extension, the humeroradial (HR) articulation enables axial rotation, and the radioulnar articulation allows pronation and supination. ${ }^{12}$ In addition to these movements, the elbow has to work as a fulcrum for the forearm lever and as a weightbearing joint. ${ }^{23}$ To fulfil these functions the elbow must be free from pain, mobile, and stable. $^{3}$ Destruction of the elbow in rheumatoid arthritis (RA) may result in considerable functional disability in the arm. ${ }^{4}$

Half of the patients with RA have elbow destruction..$^{5-7}$ Plain radiographs of the elbow will continue to be the primary diagnostic means of evaluating the $\mathrm{HU}$ and $\mathrm{HR}$ joints in the course of rheumatoid destruction. ${ }^{389}$ Subluxation of the elbow has been documented as a radiological finding in the late stage of the elbow involvement. ${ }^{10}$ Both proximal migration of the ulna and anterior subluxation of the head of the radius have been described. ${ }^{3} 5^{11-13}$ Radial head excision has been shown to destroy normal load sharing action between the HU and HR joints, and lateral instability has also been clinically documented as a consequence of this procedure. ${ }^{414}{ }^{15}$ In addition, completely flail joint due to gross bone destruction has been described as an end stage finding. ${ }^{311}$

These previously described findings are based on visual evaluation of radiographs and, to our knowledge, no measurements from radiographs have been previously published. This study aimed at measuring and analysing the HU angle and HU and HR subluxations in a cohort of 74 patients with RA followed up for 15 years.

\section{Materials and methods}

During the period $1973-75$ a total of 121 patients with recent ( $<6$ months) RA were studied at the Rheumatism Foundation Hospital, Heinola, Finland. The selection criteria, data collection strategy, and details of the patients are described elsewhere. ${ }^{16}{ }^{17}$ At the three year follow up 102 patients had rheumatoid factor positive and erosive RA. Subsequently, 24 patients died, and four patients failed to attend the 15 year follow up. Thus 74 patients ( 18 men, 56 women) were the subjects of the present study; the age at onset ranged from 17 to 66, mean 42 (SD 12) years.

Radiographs of all the 148 elbows, including also those of patients who had had previous surgery, were taken at the 15 year follow up as part of a radiographic survey study. The following standard positioning was used for the 


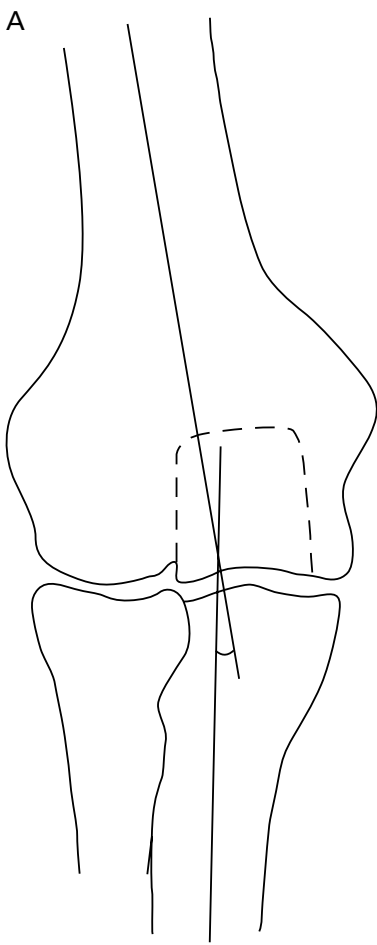

B

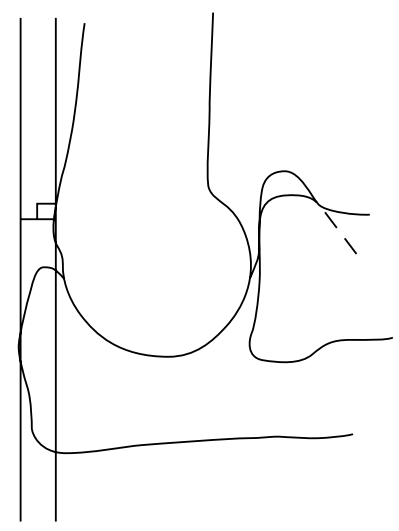

C

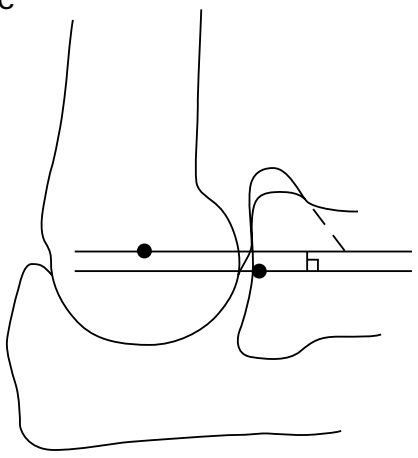

Figure 1 Schematic illustration of the measurement methods used in this study. (A) Humeroulnar (HU) angle; (B) HU proximal subluxation; (C) humeroradial anteroposterior subluxation. See "Materials and methods" for further details.

anteroposterior $(\mathrm{AP})$ view: patient sitting with shoulder in 90 degrees flexion and elbow extended in 180 degrees-or maximal extension - on the examination table, the hand supinated, the radius and the ulna in the same plane, palm facing upwards. The following standard positioning was used for the lateral view: patient sitting with shoulder in 90 degrees abduction and elbow in 90 degrees flexion on the examination table, the hand supinated, thumb facing upwards. The same radiographer confirmed the correct positioning and took the radiographs using a standard technique: The straight projections (distance $0.95 \mathrm{~m}$ ) were used with exposure factors of 45 $\mathrm{kV}$ and $-50 \mathrm{~mA}$. The size of the film (Agfa Scopix) was $18 \times 24 \mathrm{~cm}$.

The HU angle was measured from AP radiographs as the angle between the midlines of the shafts of the humerus and the ulna. The midlines were defined with a ruler. The measuring site was the crossing point of the lines in the middle of the articulation (fig 1A). Valgus was considered positive, whereas varus was negative. The HU proximal subluxation was measured from lateral radiographs as the tangential distance between the most posterior surfaces of the humerus at the site of the upper rim of the trochlea and the olecranon of the ulna (fig 1B). The line according to the humerus defined the zero point, and values posterior to this were considered positive. The HR AP subluxation was measured from lateral radiographs as the tangential distance between the midpoint of the articular surface of the capitellum and the midpoint of the articular surface of the head of the radius (fig 1C). The midpoint was defined with a ruler. The midline according to the humerus defined the zero point, and values anterior to this were considered positive.
The elbow joints were classified according to the standard reference films of six Larsen grades from 0 to $5 .{ }^{18}$ Ten synovectomies and one excision arthroplasty had been performed in nine elbows of seven patients (two bilateral synovectomies). Excision of the head of the radius had been combined with synovectomy in eight elbows. One elbow had undergone resynovectomy and one elbow excision arthroplasty after synovectomy. No total elbow replacement had been performed in these patients. The HR AP measurement was excluded in the eight elbows with resection of the head of the radius and in one totally destroyed Larsen grade 5 elbow which had no preserved anatomical contours.

Relations were estimated by Spearman's non-parametric correlation coefficient method. Age and sex adjusted means of HU angle values for Larsen grades were estimated with a regression model.

\section{Results}

There was no systematic right-left difference in either sex and, therefore, measurements of the two sides were pooled. The mean HU angle in 148 rheumatoid elbows was $11.5^{\circ}$ (SD 6.1), range $-21^{\circ}$ (varus) to $34^{\circ}$ (valgus); $9.9^{\circ}$ (SD 4.3 ) in men and $12.0^{\circ}$ (SD 6.4) in women. The mean angle of the right elbows was $11.6^{\circ}$ (SD $7.1)$ and the mean of the left side was $11.5^{\circ}$ (SD 4.8).

Table 1 shows the distribution of the 148 elbows according to the Larsen grading and the mean HU angle for each group. The nine Larsen grade 5 joints had a range of $-21^{\circ}$ to $24^{\circ}$, including all the three negative (varus) values of all the elbows. The mean HU angle of the affected joints (Larsen grades 2-4, n=66) was $14.4^{\circ}(\mathrm{SD} 6.0)$, range $0-34^{\circ}$, when totally destroyed Larsen 5 joints were excluded. The 
Table 1 Radiographic assessement of 148 rheumatoid elbows according to the Larsen grading and mean humeroulnar (HU) angle and HU joint proximal subluxation measurements for each group

\begin{tabular}{lccc}
\hline $\begin{array}{l}\text { Larsen } \\
\text { grade }\end{array}$ & $\begin{array}{l}\text { Number of } \\
\text { joints (\%) }\end{array}$ & $\begin{array}{l}\text { HU angle } \\
\text { Mean (SD) }\end{array}$ & $\begin{array}{l}\text { HU subluxation, mm } \\
\text { Mean (SD) }\end{array}$ \\
\hline 0 & $36(24)$ & $9.2(1.8)$ & $-1.4(1.6)$ \\
1 & $37(25)$ & $10.3(2.9)$ & $-0.8(1.3)$ \\
2 & $49(33)$ & $13.4(4.1)$ & $0.6(1.6)$ \\
3 & $13(9)$ & $15.2(7.4)$ & $2.2(1.8)$ \\
4 & $4(3)$ & $24.5(11.7)$ & $5.8(4.5)$ \\
5 & $9(6)$ & $4.4(13.0)$ & $7.9(6.8)$ \\
All & $148(100)$ & $11.5(6.1)$ & $0.5(3.3)$ \\
\hline
\end{tabular}

mean of the non-affected (Larsen grades $0-1$, $\mathrm{n}=73$ ) joints was $9.8^{\circ}$ (SD 2.5), range $5-17^{\circ}$. The mean HU angle of the joints with the head of the radius resected $(n=8)$ was $12.3^{\circ}$ (SD 14.2). However, this group had the most deformed Larsen 5 joint with a $21^{\circ}$ varus angle. If this joint was excluded the mean of the resected joints $(n=7)$ was $17.0^{\circ}$, range $11-24^{\circ}$. Figure 2 shows all the HU angle values with age and sex adjusted mean values (95\% confidence interval) according to the Larsen grades of the elbows.

Mean HU subluxation in 148 rheumatoid elbows was $0.5 \mathrm{~mm}$ (SD 3.3), range -4 to 21 $\mathrm{mm} ; 0.1 \mathrm{~mm}$ (SD 2.3) in men and $0.6 \mathrm{~mm}$ (SD $3.5)$ in women. Table 1 shows the distribution of the elbows ( $n=148)$ according to the Larsen grading and the mean HU subluxation for each group. Mean HU subluxation, $2.0 \mathrm{~mm}$ (SD 3.8), range -4 to $21 \mathrm{~mm}$, of the affected joints (Larsen $2-5, \mathrm{n}=75$ ) was greater than the mean, $-1.1 \mathrm{~mm}$ (SD 1.5), range -4 to $3 \mathrm{~mm}$, of the non-affected (Larsen $0-1, \mathrm{n}=73$ ) joints. Mean HU subluxation of Larsen grade $3-5$ joints $(\mathrm{n}=26)$ was $4.7 \mathrm{~mm}$ (SD 5.1). The HU proximal subluxation correlated with the increasing destruction (Larsen grading) of the elbow joint, $r_{\mathrm{s}}=0.64(95 \%$ CI 0.53 to 0.73$)$.

Mean HR subluxation in 139 rheumatoid elbows was $0.2 \mathrm{~mm}$ (SD $1.4 \mathrm{~mm}$ ), range -2 to

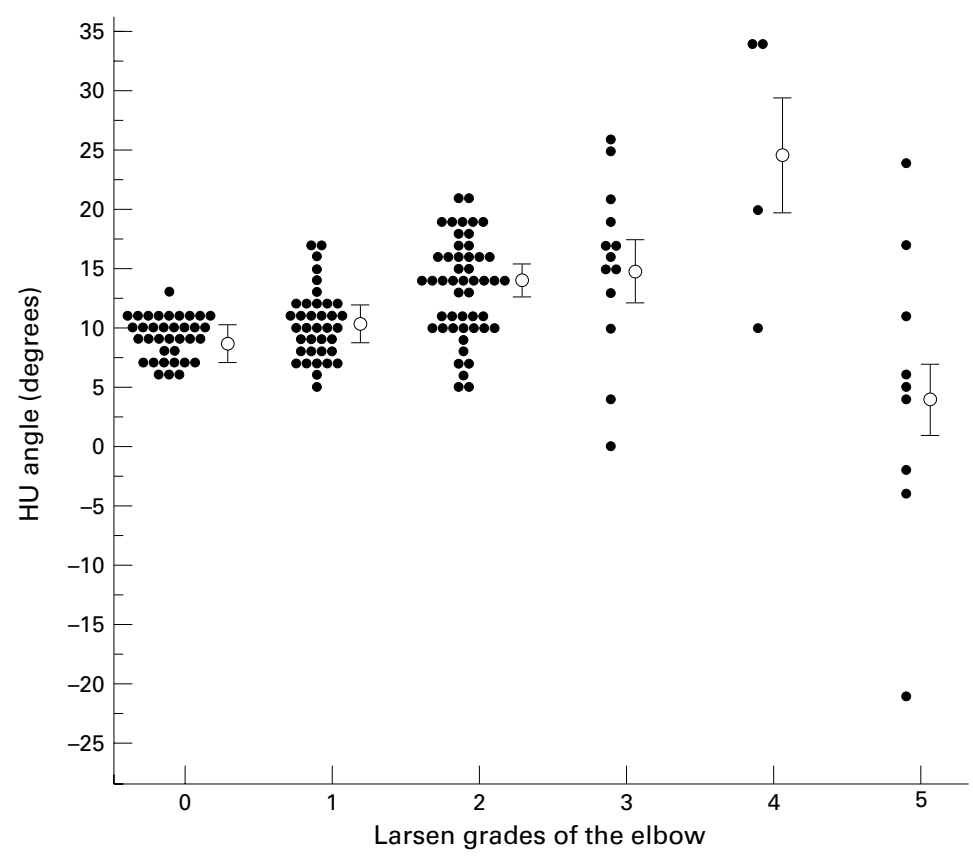

Figure 2 Humeroulnar (HU) angle values with age and sex adjusted mean values (95\% confidence interval) according to the Larsen grading of 148 rheumatoid elbows.
Table 2 Radiographic assessement of 139 rheumatoid elbows according to the Larsen grading and mean humeroradial (HR) anteroposterior subluxation measurements for each group

\begin{tabular}{lcc}
\hline Larsen grade & $\begin{array}{l}\text { Number of } \\
\text { joints (\%) }\end{array}$ & $\begin{array}{l}\text { HR anteroposterior } \\
\text { subluxation, mm } \\
\text { Mean (SD) }\end{array}$ \\
\hline 0 & $36(26)$ & $-0.4(0.9)$ \\
1 & $37(27)$ & $-0.3(0.9)$ \\
2 & $48(35)$ & $0.3(1.0)$ \\
3 & $10(7)$ & $2.0(2.1)$ \\
4 & $3(2)$ & $0.7(2.1)$ \\
5 & $5(4)$ & $3.0(1.4)$ \\
All & $139(100)$ & $0.2(1.4)$ \\
\hline
\end{tabular}

$6 \mathrm{~mm} ; 0.1 \mathrm{~mm}$ (SD 1.1) in men and $0.2 \mathrm{~mm}$ (SD 1.4) in women. Table 2 shows the distribution of the elbows $(n=139)$ according to the Larsen grading and the mean HR subluxation for each group. The mean HR subluxation, $0.8 \mathrm{~mm}$ (SD 1.5), range -1 to 6 $\mathrm{mm}$, of the affected joints (Larsen $2-5, \mathrm{n}=66$ ) was greater than the mean, $-0.4 \mathrm{~mm}$ (SD 0.9), range -2 to $2 \mathrm{~mm}$, of the non-affected (Larsen $0-1, n=73$ ) joints. Mean HR subluxation of Larsen grade $3-5$ joints $(n=18)$ was $2.1 \mathrm{~mm}$ (SD 2.0). The HR anterior subluxation correlated with the destruction of the elbow joint, $r_{\mathrm{s}}=0.48(95 \%$ CI 0.34 to 0.60$)$. The HR anterior subluxation correlated also with the $\mathrm{HU}$ subluxation of the elbow joint, $r_{\mathrm{s}}=0.37(95 \%$ CI 0.22 to 0.50$)$.

\section{Discussion}

Different methods of evaluation have been developed for radiographic assessment of rheumatoid elbow joints, the Larsen method being the most widely used. ${ }^{1011} 1418$ These radiographic classifications are based on bone erosion, not on positional changes of the three bones constituting the elbow. However, most radiological reviews describe and document different positional changes due to elbow destruction in rheumatoid arthritis; these reports all being based on visual observations. ${ }^{13} 5$ 10-13

As a first positional change before marked bone destruction most authors describe anterior, anterolateral, or ventral subluxation of the head of the radius in relation to the capitellum of the humerus. ${ }^{5}{ }^{12} 13$ In our patients the normal or non-affected (Larsen 0 and 1) elbows had the radius quite even, or a little lower in relation to the capitellum of the humerus. There was a slight tendency towards anterior subluxation of the radius in the affected joints. However, owing to excision in eight and severe destruction in one of the radial heads there were only three Larsen grade 4 and five Larsen grade 5 joints available for this measurement. Although there might have been some anterior positioning of the radial head in earlier evaluations, it might also have been due to a non-standard radiograph. We had a standardised method for the 15 year follow up radiographs, with the forearm supinated. However, to evaluate the phase of destruction some previous radiographs of our patients taken by clinical indication were also evaluated and it seemed obvious that without full supination of the hand the radial head seemed to locate more anteriorly in relation to the humerus. 

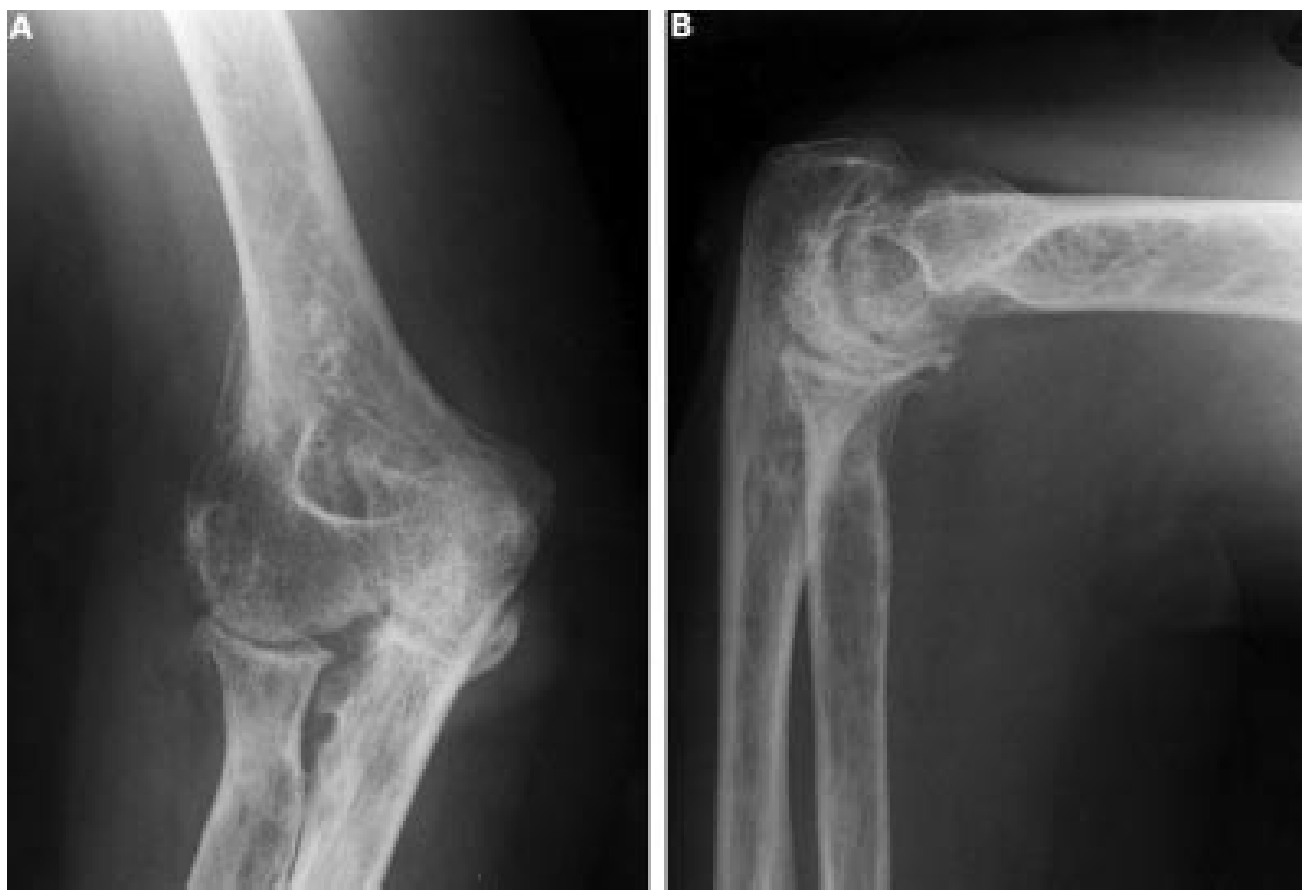

Figure 3 (A) Anteroposterior and (B) lateral radiograph of a rheumatoid elbow (Larsen grade 4). Valgus angulation has occurred with slight proximal subluxation of the ulna.

Hastings and Kwok described the anteriorly subluxated radial head as acting as a mechanical block for extension and flexion once there was erosive change in the trochlea. ${ }^{13}$ In addition to this interference with free movements, Gschwend has noted that this ventral subluxation produces a defect in the capitellum of the humerus. ${ }^{5}$ This was not seen in any of our patients. According to these results it can be stated that when the standard method of radiography described here is used, the normal joint should have the midpoint of the surfaces on the same level. Whenever anterior subluxation $(>2 \mathrm{~mm})$ is present this may be a sign of rheumatoid involvement and a cause of functional disability of the elbow.

According to Hastings and Kwok joint space narrowing and marginal erosions are followed by destruction of the HU joint. ${ }^{13}$ Souter describes further trochlear destruction as leading to gradual proximal migration of the olecranon into the residual fork formed by supracondylar ridges of the humerus. ${ }^{311}$ Our findings support these positional changes during rheumatoid elbow destruction. The posterior margin of the olecranon of the nonaffected joints (Larsen 0 and 1 ) seems to locate anteriorly or distally in relation to the humerus. Affected joints had the olecranon posteriorly in relation to the humerus and this proximal migration increased gradually during elbow destruction, leading to almost $1 \mathrm{~cm}$ difference between non-affected and severely affected (Larsen 5) joints. This proximal migration causes medial compression forces, which increase the risk for stress fracture of the medial ridge, leading to medial dislocation of the elbow, the joint becoming totally flail. ${ }^{311}$

Although this kind of fracture was not seen in our patients, the risk for such a complication was evident in those affected joints with proximal migration of the ulna. Souter states that appropriate surgical intervention at stages 3, 4, and 5 (modified from Larsen) should completely avert this catastrophe. ${ }^{11}$ Larsen grade of 3,4 , or 5 destruction has nowadays been used as a basis for considering arthroplasty for a painful elbow joint. ${ }^{3619}$ Also, according to our results this group (Larsen 3-5) has proximal migration of the ulna, and therefore orthopaedic consultation at Larsen grade 3 at the latest should be encouraged to avoid difficulties in surgery of the very complicated cases.

The HU angle of the non-affected joints, approximately 10 degrees, shows that there is a slight valgus angle in normal elbows with a standard method, as reported previously. ${ }^{20}$ Rheumatoid involvement of the elbow seems to increase valgus angulation. This deformity increases according to Larsen grading, ending up with a mean valgus at grade 4 almost three times as great as that with the normal position (fig 3). However, at grade 5 the mean angle collapses into a more neutral position, even under the normal variation in these elbows. This can be explained by the gross bone destruction in these joints, which has been described as leading to complete disruption of the ligaments and dislocation of the joint. ${ }^{1012}$ This is supported by the fact that only varus angles and widest range (45 degrees) of this material were measured within the Larsen grade 5 group. When this unstable group was excluded the affected joints had evidently more valgus than the non-affected joints and this phenomenon progressed gradually during destruction.

The timing and type of synovectomy in treatment of the rheumatoid elbow has been disputed. $^{3521}$ Most authors support radial 
head resection as an important part of the procedure. $^{314}$ Radial head excision destroys the normal load sharing action, concentrating force on the coronoid and requiring medial collateral ligament tension to prevent valgus deformity. ${ }^{4}$ Replacement of the radial head with a silicone prosthesis has been largely abandoned because early breakage and wear debris initiated synovitis. ${ }^{22}$ In these elbows the excision of the radial head seemed to have a valgus provoking effect. The valgus of the joints which had undergone this procedure was twofold greater than that of non-affected joints and greater than that of affected joints as well. However, the material has only seven evaluable (one destroyed) cases without any preoperative measurements, so further studies of this issue are needed.

We conclude that slight anterior subluxation of the radius with progressive proximal migration and subluxation of the ulna are consequences of rheumatoid elbow destruction. In addition, the HU angle turns into valgus, which may be provoked by radial head resection, during rheumatoid changes. Larsen grade 5 elbows seem to have severe bone deficiency and instability with a high risk for complications, and therefore orthopaedic consultation should be performed at an earlier stage.

1 Forrester DM, Brown JC, eds. The elbow. In: Radiology of joint disease. New York: Saunders, 1987:318-49.

2 Lewis G. The elbow joint and its total arthroplasty. Part I. A state-of-the-art review. Biomed Mater Eng 1996;6:353-65.

Souter WA Surgery of the rheumatoid elbow. Ann Rheum Dis 1990;49:871-82.

4 Amis AA, Hughes SJ, Miller JH, Wright V. A functional study of the rheumatoid elbow. Rheumatol Rehabil study of the rhe
$1982 ; 21: 151-7$.
5 Gschwend N. Operations in the region of the elbow joint. In: Surgical treatment of rheumatoid arthritis. New York: Thieme, 1980:45-66.

6 Ljung P, Jonsson K, Rydgren L, Rydholm U. The natural course of rheumatoid elbow arthritis: a radiographic and clinical five-year follow-up. Journal of Orthopedics and Rheumatology 1995;8:32-6.

7 Lehtinen JT, Kaarela K, Ikävalko M, Kauppi MJ, Belt EA, Kuusela PP, et al. Incidence of elbow involvement in rheumatoid arthritis. A 15 year endpoint study. J Rheumatol 2001;28:70-4.

8 Kaye JJ. Arthritis: roles of radiography and other imaging techniques in evaluation. Radiology 1990;177:601-8.

9 Scutellari PN, Orzincolo C. Rheumatoid arthritis: sequences. Eur J Radiol 1998;27:S31-5.

10 De Sèse S, Debeyre N, Diian A, Manuel R. The elbow joint. In: Carter ME, ed. Radiological aspects of rheumatoid arthritis. Nijmegen: F E MacDonald, 1964:115-36.

11 Souter W A. Surgery for rheumatoid arthritis: upper limb surgery of the elbow. Current Orthopaedics 1989;3:9-13.

2 Berens DL, Ru-Kan L, eds. Elbow. In: Roentgen diagnosis of rheumatoid arthritis. Springfield: Thomas, 1969:232-44.

13 Hastings DE, Kwok J. Rheumatoid elbow deformity. J Bone Joint Surg Br 1982;64:634.

14 Stein H, Dickson RA, Bentley G. Rheumatoid arthritis of the elbow. Pattern of joint involvement, and results of synovectomy with excision of the radial head. Ann Rheum Dis 1975;34:403-8.

15 Resnick D, ed. Diagnosis of bone and joint disorders. Rheumatoid arthritis. 3rd ed. Philadelphia: Saunders; 1988:896.

16 Kaarela K. Prognostic factors and diagnostic criteria in early rheumatoid arthritis. Scand J Rheumatol 1985;14(suppl 57): $1-54$.

17 Kaarela K, Kautiainen H. Continuous progression of radiological destruction in seropositive rheumatoid arthritis. J Rheumatol 1997;24:1285-7.

18 Larsen A, Dale K, Eek M. Radiographic evaluation of rheumatoid arthritis and related conditions by standard reference films. Acta Radiol Diagn 1977;18:481-91.

19 Ljung P, Jonsson K, Larsson K, Rydholm U. Interposition arthroplasty of the elbow with rheumatoid arthritis. J Shoulder Elbow Surg 1996;5:81-5.

20 Scales JT, Lettin AW, Bayley I. The evolution of the Stanmore hinged total elbow replacement. In: foint replacement of the upper limb. London: Inst. Mech. Engl. Publ., 1977:53-62.

21 Taup NJ, Winia WPCA. Synovectomy of the elbow in rheumatoid arthritis. J Bone Joint Surg Br 1989;71:664-6.

22 Trepman E, Ewald FC. Early failure of silicone radial head implants in the rheumatoid elbow. J Arthroplasty 1991;6: 59-65. 